\title{
Biomechanical analysis of the circular friction hand massage
}

\author{
Jeseong Ryu, Jongsang Son, Soonjae Ahn, Isu Shin and Youngho Kim* \\ Department of Biomedical Engineering and Institute of Medical Engineering, Yonsei University, \\ Wonju-si, Gangwon-do, Korea
}

\begin{abstract}
.
BACKGROUND: A massage can be beneficial to relieve muscle tension on the neck and shoulder area. Various massage systems have been developed, but their motions are not uniform throughout different body parts nor specifically targeted to the neck and shoulder areas.

OBJECTIVE: Pressure pattern and finger movement trajectories of the circular friction hand massage on trapezius, levator scapulae, and deltoid muscles were determined to develop a massage system that can mimic the motion and the pressure of the circular friction massage.

METHODS: During the massage, finger movement trajectories were measured using a 3D motion capture system, and finger pressures were simultaneously obtained using a grip pressure sensor.

RESULTS: Results showed that each muscle had different finger movement trajectory and pressure pattern. The trapezius muscle experienced a higher pressure, longer massage time (duration of pressurization), and larger pressure-time integral than the other muscles.

CONCLUSIONS: These results could be useful to design a better massage system simulating human finger movements.
\end{abstract}

Keywords: Circular friction hand massage, finger pressure, motion analysis

\section{Introduction}

Technological advancements have drastically decreased the physical activity among people. The consequent decrease of muscle mass and quantity might have caused cramps, stiffness, and sometimes adhesive capsulitis in muscles. In particular, people working in the office with insufficient exercise frequently experience pain in their neck and shoulders due to the muscle contraction caused by chronic stress [1]. Exercises such as stretching or massage are beneficial to relieve the muscle tension, and professional help (i.e., expert massage or physical therapy) might be also needed in case of severe pain.

Studies in the early 90's revealed that massage directly stimulates blood circulation in muscles, skin, and cartilage [2 -6]. Moreover, studies during the late 90's showed that massage assisted rehabilitation by stimulating human body's metabolism and removing body wastes [7--10]. Cafarelli and Flint [11] also suggested that massage stimulates the peripheral nerves of muscles and thus improves its recovery by increasing blood and lymph circulation.

\footnotetext{
${ }^{*}$ Corresponding author: Youngho Kim, Department of Biomedical Engineering and Institute of Medical Engineering, Yonsei University, Wonju-si, Gangwon-do 220-710, Korea. Tel.: +82 33760 2492; E-mail: younghokim@ yonsei.ac.kr. 

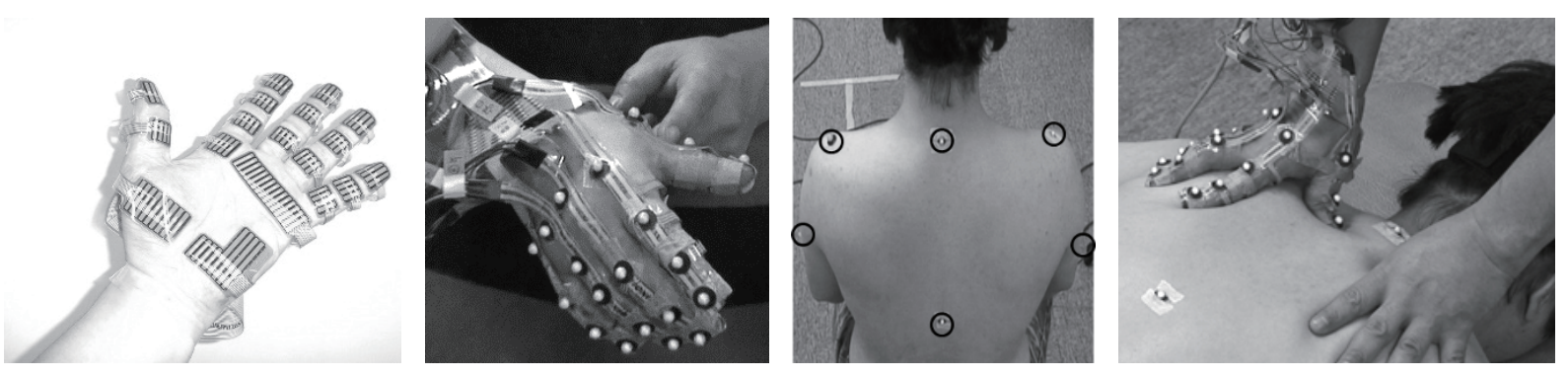

Fig. 1. Experimental setting with grip pressure sensor and reflective markers.

Recently, well-being has been considered as a high priority. To satisfy the increased demand for home health machines and massage systems, lately developed devices imitate motions of hand massage; however, their motions are not uniform throughout different body parts nor specifically targeted to the neck and shoulder areas. Though massage produces positive effects in blood circulation, stimulation, relaxation, reflex, and congest [12], mechanical massage systems are less effective than professional hand massage and sometimes result in painful suffering.

Typical massage techniques are based on effleurage (stroking), friction, petrissage (kneading), vibration, tapotement (hacking, tapping, clapping, and beating), rolling and so on. They can be further divided into 23 methods considering variations of intensity and area of application. However, existing massage systems utilize friction, tapotement, and vibration only, thus their effects are limited in comparison with the professional hand massage. Especially, sports massage generally adopts the circular friction massage technique [12[13]. In this study, hand motion and finger pressure were simultaneously measured during the circular friction massage on trapezius, levator scapulae, and deltoids near neck and shoulders areas. Then, movement trajectories and pressure distribution were compared among those muscles.

\section{Subjects and methods}

\subsection{Subjects}

A professional male sports masseur, an expert in the circular friction massage, participated in this study. Five males were recruited, who showed no sign of injury or pathological diagnosis on their upper bodies. Their age, mass, and height were $27.5 \pm 4.3$ years, $73.4 \pm 8.6 \mathrm{~kg}$, and $173.3 \pm 4.1 \mathrm{~cm}$, respectively. The subjects were prone while the masseur used his weight to apply sufficient pressure during the massage. The masseur practiced sufficiently before the actual measurements. All participants provided their informed written consents which were approved by the Institutional Review Board of Yonsei University.

\subsection{Experimental methods}

A grip pressure sensor and reflected markers were attached to the masseur's hand with double-sided tapes. Based on the marker set of Zhang et al. [14], as shown in Fig. 1, nineteen spherical reflective markers of $6.4 \mathrm{~mm}$ diameter were attached on finger joints and on the top of the grip sensors at fingertips. Two markers at the wrist were excluded, since the wrist motion is minimal in the circular friction massage. In addition, reflective markers were placed to C7, T10, CLAV, LSHO, LUPA, RSHO, and RUPA (Fig. 1 
Table 1

Additional reflective markers in the upper body

\begin{tabular}{lll}
\hline Abbreviation & Full name & Position \\
\hline C7 & 7th cervical vertebrae & Spinous process of the 7th cervical vertebrae \\
T10 & 10th thoracic vertebrae & Spinous process of the 10th thoracic vertebrae \\
CLAV & Clavicle & Jugular notch where the clavicles meet the sternum \\
LSHO & Left shoulder & Placed on the acromioclavicular joint \\
LUPA & Left upper arm & Place on the upper arm between elbow and shoulder markers \\
RSHO & Right shoulder & Placed on the acromioclavicular joint \\
RUPA & Right upper arm & Place on the upper arm between elbow and shoulder markers \\
\hline
\end{tabular}

Table 2

Trajectories for different locations based on ellisoids

\begin{tabular}{lccccc} 
& & & & $(\mathrm{mm})$ \\
\hline Long-axis & Trapezius & Levator scapulae & Anterior deltoid & Middle deltoid & Posterior deltoid \\
Short-axis & $9.0 \pm 2.3$ & $14.0 \pm 1.1$ & $12.8 \pm 2.4$ & $6.5 \pm 1.3$ & $8.2 \pm 1.0$ \\
Ratio (L/S) & $1.32 \pm 0.16$ & $13.7 \pm 1.2$ & $11.0 \pm 3.1$ & $4.9 \pm 2.1$ & $6.6 \pm 1.7$ \\
\hline
\end{tabular}

*Ratio (L/S) means ratio between long-axis and short-axis.

and Table 1) to determine massage locations in the upper body. The marker trajectories were recorded using the 3D motion capture system (Vicon MX system, Vicon Motion System, Ltd, UK) with 10 infrared cameras (Vicon MX-T40) at a sampling rate of $120 \mathrm{~Hz}$. Pressure distributions were measured using a grip pressure sensor (Grip EDGE 4256E, Tekscan, Inc., USA) and cuff (Versatek Cuff, Tekscan, Inc., USA). The dimensions of the grip pressure sensor was $1.6 \mathrm{~cm} \times 1.6 \mathrm{~cm}$ on the tip of the thumb.

The target of the circular friction massage consisted of 17 points: 3 points on levator scapulae, 5 points on trapezius, and 9 points on deltoid. The massage was continuously applied 6 times to each point. Both pressing and releasing motions were averaged every cycle. Assuming that the other fingers except the thumb provide only support to the hand but less massage effect, only trajectories of the thumb were analyzed in this study.

\subsection{Statistical analysis}

One-way ANOVA test was performed to compare peak pressures and durations of five different massage locations with Tukey's HSD post hoc test. All statistical analyses were performed with IBM SPSS Statistics (Version 20, IBM, USA), and the significance level ( $p$ ) was set at 0.05 .

\section{Results}

\subsection{Massage motion}

The trajectories of the thumb looked like an ellipsoid around the massage area. Trajectories in trapezius and middle deltoid resembled more an ellipsoid, but trajectories in levator scapulae were close to a circle. The masseur used different massage skills to different locations. Since trapezius muscle is stiffer than the other muscles, the masseur moved his thumb more strongly along the muscle fiber direction. For levator scapulae, however, he moved the thumb more softly in a circular shape to avoid subjects to get pain. Table 2 represents the marker trajectories for different locations based on ellipsoids (Table 2). 
Table 3

Relationship between peak pressure and time for different locations

\begin{tabular}{lrcccc}
\hline & \multicolumn{1}{c}{ Trapezius } & Levator scapulae & Anterior deltoid & Middle deltoid & Posterior deltoid \\
\hline Pressure $(\mathrm{kPa})$ & $124.52 \pm 19.55$ & $74.53 \pm 11.06$ & $82.15 \pm 5.34$ & $75.66 \pm 6.68$ & $76.98 \pm 4.22$ \\
Duration $(\mathrm{sec})$ & $1.18 \pm 0.32^{*}$ & $0.73 \pm 0.11 \dagger$ & $0.91 \pm 0.04$ & $0.80 \pm 0.07$ & $0.75 \pm 0.05$ \\
\hline
\end{tabular}

$*, \dagger$ Significantly different from the other four muscles $(p<0.05)$.

Table 4

Pressure-time integral (PTI) for different massage locations

\begin{tabular}{lcccc} 
& & & $(\mathrm{kPa} . \mathrm{sec})$ \\
\hline Trapezius & Levator scapulae & Anterior deltoid & Middle deltoid & Posterior deltoid \\
\hline $92.8 \pm 24.0^{*}$ & $39.0 \pm 8.9 \dagger$ & $57.4 \pm 11.7$ & $51.0 \pm 8.0$ & $55.3 \pm 3.9$ \\
\hline
\end{tabular}

$*, \dagger$ Significantly different from the other four muscles $(p<0.05)$.

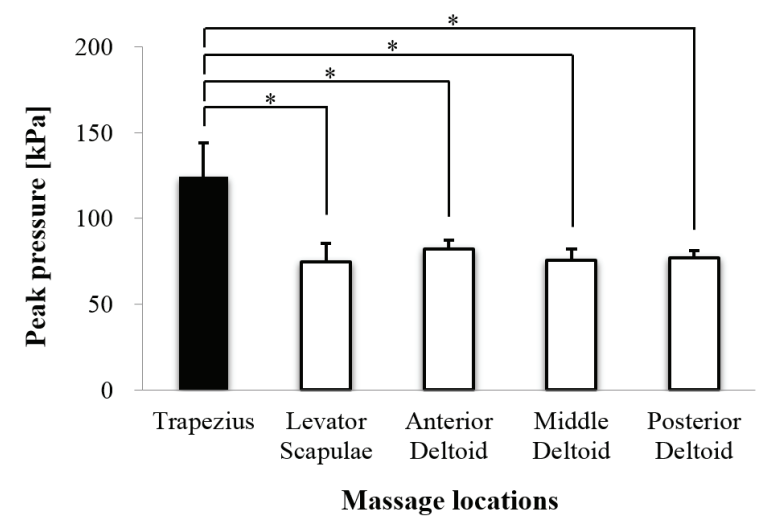

(a) in pressing

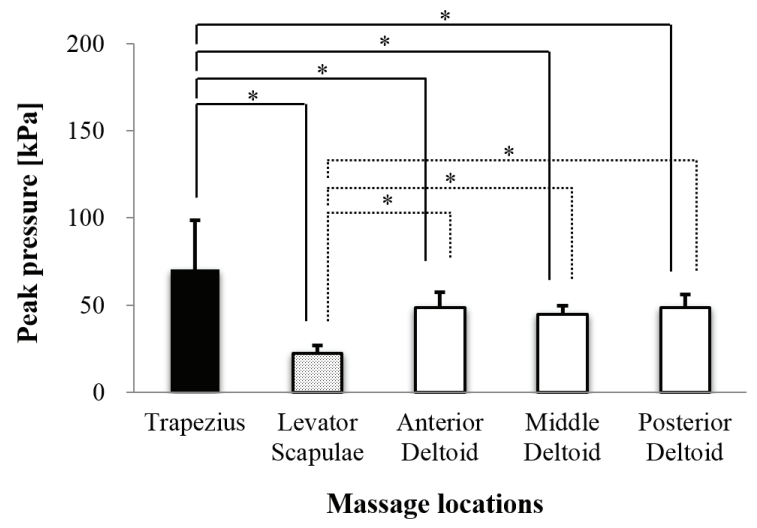

(b) in releasing

Fig. 2. Peak pressure for different massage locations, *significant difference with $p<0.05$.

\subsection{Massage pressure}

The peak pressure depended on the location of the massage. Figure 2 shows peak pressures for different massage locations. Significantly high pressure was observed in trapezius than in the other locations. Levator scapulae showed significantly low peak pressure than the other locations.

The duration (time) of each massage cycle depended on the location of the massage. Longer massage time was noted in trapezius than in the other locations. The massage time of levator scapulae showed significant difference from anterior and middle deltoids. No significant difference in the massage time for each cycle was found between anterior and middle deltoids. In addition, massage time correlated positively with the peak pressure $\left(r^{2}=0.93\right)$; higher pressure with longer massage time (Table 3 ).

Table 4 represents the pressure-time integral (PTI) for different massage locations. The trapezius showed the largest PTI, but levator scapulae showed the smallest.

\section{Discussion and conclusion}

The circular friction massage on trapezius, deltoid, and levator scapulae near the neck and shoulders 
resulted in different pressure patterns and movement trajectories among different muscles. To patients with myofascial pain syndrome at trapezius and levator scapulae, Fernández et al. [15] applied the ischemic compression technique and transverse friction massage at the trigger point of each patient with pressures tantamount to the pressure pain threshold. Then, they assessed the patient's pain level according to the visual analogue scale, and found that it had significantly decreased from 4.9 to 0.7 . Their study demonstrated the efficacy of the massage by conducting various types of massage with predetermined pressures to patients. In contrast, this study targeted healthy subjects to acquire the magnitude of pressure, duration of pressurization, and motion data of each point in three muscle groups. Lee et al. [16] evaluated the period and the magnitude of pressurization trials by using thin pressure sensors attached to fingers. In contrast, the finger motion analysis and quantitative massage pressure measurements were more systematically applied in the present study.

The resultant trajectory of the thumb's tip looked like an ellipse. The ratios of the long-axis to the short-axis of the elliptical trajectories were similar within points on the same locations. This means that the masseur's hand motion is differed by the structure of the muscle. The pressure and motion of the massage were dependent on the posture and the instant feedback of both the subject and the masseur. Consequently, masseurs would vary pressure, motion, and the number of repetition in response to the subject's instant feedback. Therefore, to acquire a generalized pattern for the massage, ample discussion with the masseur was made prior to the trials to apply motions and pressures based on the masseur's experience. The circular friction method, prevalent in hand massages, utilizes only the thumb tip. Therefore, the shape and area of the tip of the massage might be important factors, since those are related with massage pressure.

In summary, massage motion, which is the trajectory of the masseur's thumb, looked like an ellipsoid in all muscles except levator scapulae. Long-axis of finger movement trajectories were along the muscle fiber direction. Trajectories in the levator scapulae were close to a circle. Peak pressure on trapezius was higher than the other muscles, but levator scapulae had significantly low pressure among all muscles in releasing pressure. Also, trapezius showed significantly long duration (time) of each massage cycle and large pressure-time integral. Levator scapulae showed significantly short duration of each massage cycle and small pressure-time integral. The shape and the area of the tip of the massage might be important factors in the circular friction massage. These results could be useful to develop a massage system that can mimic the motion and the pressure of the circular friction massage.

\section{Acknowledgement}

This research was financially supported by Dae Kyoung Ind. Co., LTD. through the massage chair project (No. 2010-8-5280).

\section{References}

[1] Kim YD, Choi DH, Chang YS, Lee HJ, Tae KS, Massage therapy of electrical chair-massager effects on EEG patterns. J Digital Contents Society. 2010; 10(6): 364-372.

[2] Nosaka K, Clarkson PM. Relationship between post- exercise plasma CK elevation and muscle mass involved in the exercise. Int J Sports Med. 1992; 13(6): 471-475.

[3] Clarkson PM, Newham DJ. Associations between muscle soreness, damage and fatigue. Adv Exp Med Biol. 1995; 384 : 457-469.

[4] Harmer PAP. The effect of pre-performance massage on frequency in sprinters. Athletic Training. 1991; 26: 55-59. 
[5] Tiidus PM, Shoemaker JK. Effleurage massage, muscle blood flow and long term post-exercise strength recovery. Int J Sports Med. 1995; 16(7): 478-483.

[6] Ironson G, Field T, Scafidi F, Hashimoto M, Kumar M, Kumar A, et al. Massage therapy is associated with enhancement of the immune system's cytotoxic capacity. Int J Neurosci. 1996; 84(1-4): 205-217.

[7] Ernst E. Does post-exercise massage treatment reduce delayed onset. Muscle soreness? A systematic review. Br J Sport Med. 1998; 32: 212-214.

[8] Gupta S, Goswami A, Sadhukhan AK, Mathur DN. Comparative study of lactate removal in short term massage of extremities, active recovery and a passive recovery period after submaximal exercise sessions. Int J Sports Med. 1996; 17: 106-110.

[9] Smith JA, Pyne DB. Exercise, training and neutrophil Function. Exerc Immunol Rev. 1997; 3: 96-116.

[10] Weltman DL. The effects of massage on athletes' cardiorespiratory system. Soviet Sports Review. 1999; 25.

[11] Cafarelli E, Flint F. The role of massage in preparation for and recovery from exercise. An overview. Sports Med. 1992; 14(1): 1-9.

[12] Yook JY. Sports massage and body action therapy. Seoul: Geulnurim; 2010.

[13] Lee SH, Han SC, Lee SY. The effect of sports massage on stress hormone after exercise. The Korea Journal of Sports Science. 2008; 17(1): 599-607.

[14] Zhang X, Lee SW, Braido P. Determining fingers segmental centers of rotation in flexion-extension based on surface marker measurement. J Biomech. 2003; 36(8): 1097-1102.

[15] Fernández-de-las-Peñas C, Alonso-Blanco C, Fernández-Carnero J, Carlos Miangolarra-Page J. The immediate effect of ischemic compression technique and transverse friction massage on tenderness of active and latent myofascial trigger points: a pilot study. J Bodyw Mov Ther. 2006; 10(1): 3-9.

[16] Lee HM, Wu SK, You JY. Quantitative application of transverse friction massage and its neurological effects on flexor carpi radialis. Man Ther. 2009; 14(5): 501-507.

[17] James HC, David MP. Basic clinical massage therapy. NC: Lippincott Williams \& Wilkins; 2006.

[18] Lacroix N, Seager S, Rinaldi F, Tanner R, Total Body Massage, U.K.: Anness Publishing Limited; 2004. 